\title{
Formal Modeling of BeeAdHoc: a Bio-inspired Mobile Ad Hoc Network Routing Protocol
}

\author{
Muhammad Saleem ${ }^{1}$, Syed Ali Khayam ${ }^{2}$, Muddassar Farooq ${ }^{3}$ \\ ${ }^{1}$ Center for Advanced Studies in Engineering (CASE) Islamabad, Pakistan. \\ ${ }^{2}$ WisNeT, SEECS-NUST Rawalpindi, Pakistan. \\ ${ }^{3}$ nexGIN RC, NUCES-FAST Islamabad, Pakistan. \\ msaleem@case.edu.pk ${ }^{1}$ khayam@niit.edu.pk ${ }^{2}$ muddassar.farooq@nu.edu.pk ${ }^{3}$
}

\begin{abstract}
Design and development of routing protocols for Mobile Ad Hoc Networks (MANETs) is an active area of research. The standard practice among researchers working in this emerging domain is to evaluate the performance of their routing protocols in a network simulator. It is now a well known fact that the simulation studies are scenario specific and hence their results can not be generalized. In this paper, we present mathematical models of two key performance metrics, routing overhead and route optimality, of BeeAdHoc MANET routing protocol. One of the key components of our BeeAdHoc model is the collision model at Medium Access Control (MAC) layer. The mathematical expressions of the performance metrics provide valuable insight about the behavior of $B e e A d H o c$ in particular, and a typical ad hoc routing protocol in general, without resorting to scenario specific time consuming simulations.
\end{abstract}

\section{Introduction}

Design of novel routing techniques for Mobile Ad Hoc Networks (MANETs) received a significant amount of attention by researchers that resulted in a number of Bio-inspired routing protocols: AntHocNet [1], ARA [2], BeeAdHoc [3][4] and ANSI [5]. As topology of ad hoc network is dynamic, such protocols need to adapt themselves to continuously changing routes between corresponding source and destination pairs. The frequency of route changes is dependent on node density and their deployment pattern, nodes speed etc. In order to understand the behavior of protocols over a wide operational spectrum, designers create a number of different scenarios by changing the values of the above-mentioned parameters and then collect relevant performance metrics through extensive simulations that might take several days or even weeks to finish.

The simulation-based study has three shortcomings: (1) the results are scenariospecific, (2) simulation tools have limited scalability, and (3) large simulation time significantly slows down the protocol engineering cycle. Therefore, we argue that mathematical tools must be utilized to complement the simulation studies in order to overcome their shortcomings. This is also the corollary of the paper by Kurkowski et al. [6]. 
Formal modeling of MANET routing protocols is difficult due to various reasons: dynamic topology, diverse flooding patterns, contention at the MAC layer etc. In this paper, we model two well known metrics, for BeeAdHoc protocol by incorporating the collisions at the MAC layer. The derived metrics not only provide an unbiased analysis of BeeAdHoc protocol but also unveils some interesting facts about its behavior. Derived metrics may be used to derive a number of other evaluation metrics: total energy consumption, computational complexity, packet latency etc.

Related Work. The formal modeling of Bio-inspired protocols have received little attention. The exceptions are the work of Roth and Wicker [7], Saleem et al. [8] and Zahid et al. [9]. The later is however limited to fixed networks. In [7], Roth has developed an analytical framework based on the Markov chains for the analysis of probabilistic routing protocols.

Organization of Paper. The rest of the paper is organized as follows. Section 2 contains system description and modeling assumptions. A brief description of BeeAdHoc protocol is presented in section 3. Section 4 describes the modeling of routing overhead followed by the route optimality model in Section 5. Finally we conclude our paper with an outlook to our future research.

\section{System Description and Modeling Assumptions}

\subsection{Basic Graph Terminology}

A typical graph is denoted by $G(V, E)$ in which $V$ is a set of vertices in the graph and $E$ represents the set of edges. This model can be used to represent an ad hoc network in which individual nodes are the vertices of the graph connected through wireless links (or edges of the graph). In this section, definitions of some basic graph-theoretic terms are provided.

Node degree: Degree of a node $x, d(x)$, represents the number of nodes directly connected with $x$. Minimum degree of a graph $G$ is then defined as:

$$
d_{\min }(G)=\min \{d(x)\} \quad \forall \mathrm{x} \in \mathrm{G}
$$

A similar term is the average node degree defined as:

$$
d_{\text {avg }}(G)=\frac{1}{n} \sum_{x=1}^{n} d(x)
$$

Connected and disconnected graphs: A graph is connected if at least a path exists between each pair of nodes in the graph [10]; otherwise, it is a disconnected graph.

\subsection{Network Topology}

Ad hoc network topology plays the pivotal role in modeling of an upper layer protocol. Connectivity of graph depends upon the nodes deployment pattern, their 
transmission powers, environmental conditions etc. Bettstetter's seminal work [10] addresses the connectivity of wireless networks. Assuming that $N$ nodes are randomly distributed and connected through symmetric links, Bettstetter derived an expression that, for a given node density $\rho$, determines the transmission range $r_{0}$ to ensure that a randomly chosen node will have exactly $n_{0}$ neighbors. Probability $P$ that a node has exactly $n_{0}$ neighbors is given below:

$$
P\left(d=n_{0}\right)=\frac{\left(\rho \pi r_{0}^{2}\right)^{n_{0}}}{n_{0} !} \cdot e^{-\rho \pi r_{0}^{2}} .
$$

Expected or average degree of the node is $E(d)=d_{a v g}=\rho \pi r_{0}^{2}-1$. To be sure with a certain probability $p$ that the network having $n>>1$ nodes is connected,

$$
r_{0} \geq \sqrt{\frac{-\ln \left(1-p^{\frac{1}{n}}\right)}{\rho \pi}} .
$$

We can use (1) and (2) to generate the underlying network topology. Interested readers are referred to [10] for further information.

\subsection{Modeling Assumptions}

We assume a network in which $N$ nodes are deployed according to a homogeneous Poisson distribution with node density $\rho$ and an average node degree of $d_{\text {avg }}$. Each node has a transmission radius $r_{0}$ and an $802.11 \mathrm{~b}$ distributed coordination function (DCF) acts as an underlying MAC layer protocol.

\section{BeeAdHoc}

BeeAdHoc is an on-demand multi-path routing algorithm for mobile ad hoc networks inspired from the foraging principles of honey bees [4]. BeeAdHoc works with four types of agents: packers, scouts foragers and swarms. The packers locate a forager and hand over the data packet to the discovered forager. Scouts discover new routes from the launching node to the destination node through broadcasting principle and an expanding time to live (TTL) timer. Foragers, the main workers of BeeAdHoc, receive the data packets from the packers and transport them to the destination. Transportation of foragers back to the source node, in case of unreliable transport protocol, is the key role of swarms. Interested readers are referred to [4] for more details.

\section{Routing Overhead Model}

We define routing overhead as the number of scouts generated in the network up to a particular number of hops $(h)$ during a route discovery process. Our definition of routing overhead reflects the time to live mechanism employed in BeeAdHoc during a route discovery. When TTL expires, nodes stop rebroadcasting the scouts. A typical propagation pattern of scouts in BeeAdHoc is shown 


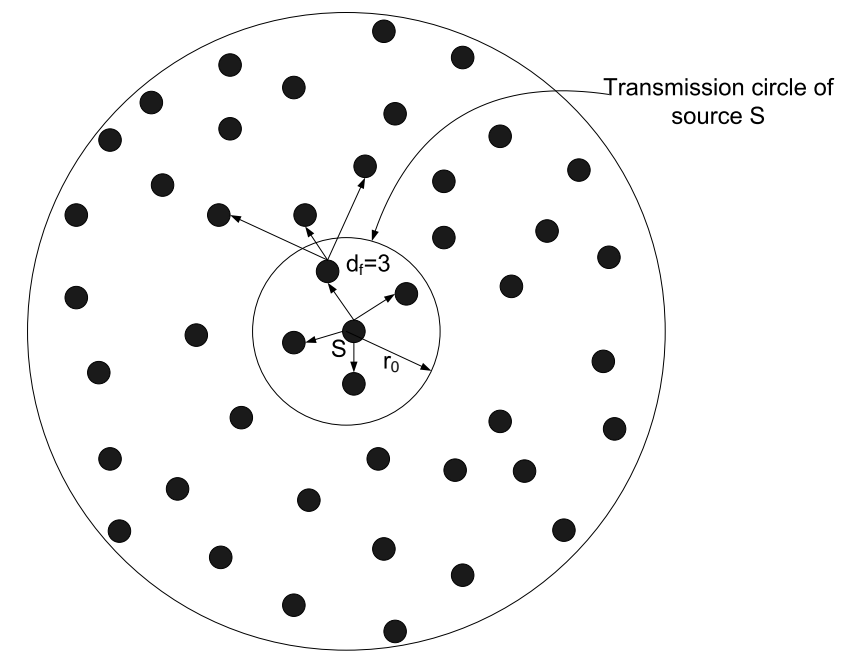

Fig. 1. Route discovery pattern in an ad hoc network

in Fig. 1 in which source $\mathrm{S}$, located at the center, broadcasts a scout to all its neighbors (i.e. nodes located within its transmission circle). In the rest of this section, we derive an expression to calculate the expected number of scouts up to $h$ hops from the source node.

\subsection{Routing Overhead in terms of Expected Forward Degree}

The first scout broadcasted by the source is received by $d_{\text {avg }}$ nodes, where $d_{\text {avg }}$ is the average degree of the node. Each one of the $d_{\text {avg }}$ neighbors rebroadcast the scout unconditionally. However, due to collisions, a fraction of the rebroadcasted scouts get lost and we do not count them in the calculation of routing overhead. Hence, the first hop rebroadcasting nodes equal $\overline{p_{c}} d_{a v g}$ where $\overline{p_{c}}$ is the probability of no collision. To compute the routing overhead of BeeAdHoc, $C_{p}^{(\text {beeadhoc })}$, we accumulate the scouts generated at each hop up to a distance of $h$ hops from the source node.

$$
\begin{aligned}
C_{p}^{(\text {beeadhoc })=} & 1+\overline{p_{c}} d_{\text {avg }}+\left(\overline{p_{c}}\right)^{2} d_{\text {avg }} \times d_{f[1]}+\left(\left(\overline{p_{c}}\right)^{3} \cdot d_{\text {avg }} \cdot d_{f[1]}\right) \times d_{f[2]}+ \\
& +\ldots+\left(\left(\overline{p_{c}}\right)^{h} \cdot d_{\text {avg }} \cdot d_{f[1]} \ldots d_{f[h-2]}\right) \times d_{f[h-1]} .
\end{aligned}
$$

Equation (3) contains a new term known as expected forward degree of a node. We define it as: "the number of new nodes that will receive the scout of that node 
and are likely to rebroadcast it to the next hop". The terms, $d_{f[1]}, d_{f[2]}, \ldots, d_{f[h-1]}$ in (3) represent the expected forward degree of nodes at $1,2, \ldots, h-1$ hops from the source node respectively. Knowing the expected forward degree of a node at a stage, we can multiply it with the number of rebroadcasting nodes at the same stage to calculate the number of nodes that are likely to rebroadcast scout at the next stage. Getting back to (3), we have a total of $h+1$ terms. Hence, its closed form is

$$
C_{p}^{(\text {beeadhoc })}= \begin{cases}1+\overline{p_{c}} d_{\text {avg }} & \text { if } h=1 \\ 1+\overline{p_{c}} d_{\text {avg }}+\overline{p_{c}} d_{\text {avg }} \sum_{i=1}^{h-1}\left(\overline{p_{c}}\right)^{i} \prod_{j=1}^{i} d_{f[j]} . & \text { otherwise }\end{cases}
$$

The above expression validates the intuition that routing overhead $C_{p}^{(\text {beeadhoc })}$ is a function of the number of hops. As the value of $h$ increases, the routing overhead increases as well. Similarly, routing overhead of BeeAdHoc varies directly with expected forward degree. On the other hand, probability of no collision has reverse effect. Theoretically, in no collision case, number of scouts must be equal to the total number of nodes within the ring. However, being a fractional term, $\overline{p_{c}}$ is likely to reduce this number substantially in dense networks.

Assuming that $d_{f[1]}=d_{f[2]}=d_{f[3]}=\ldots=d_{f[h-1]}=d_{f}$, (4) simplifies to

$$
C_{p}= \begin{cases}1+h \overline{p_{c}} d_{f} d_{\text {avg }} & \text { if } \overline{p_{c}} d_{f}=1 \\ 1+\overline{p_{c}} d_{\text {avg }}\left(\frac{1-\left(\overline{p_{c}} d_{f}\right)^{h}}{1-\overline{p_{c}} d_{f}}\right) & \text { otherwise }\end{cases}
$$

Assumption of a constant expected forward degree in (5) is valid under two scenarios. Either the network is sparse where nodes are scattered and they have no or extremely small overlapping transmission regions. Secondly, it also applies to small sized networks. With these limitations, an expression for $d_{f}$ is derived in $[8]$ and we simply reproduce it here for the sake of brevity.

$$
d_{f} \simeq \frac{d_{a v g}-\rho r_{0}^{2}\left(\frac{2 \pi}{3}-\frac{\sqrt{3}}{2}\right)}{2}
$$

Equation (6) shows that $d_{f}$ is a function of $d_{\text {avg }}$ and $r_{0}$ respectively. The concept of constant forward degree, however, does not hold for large and dense networks. As we move away from the source node, ideally expected forward degree should reduce at every step. Intuitively, as more and more nodes receive the broadcasted scouts, the uncovered area reduces. Consequently, the probability to deliver to a new area reduces and hence the expected forward degree. Apart from its dependence upon the number of hops from the source node, expected forward degree must also be marginalized i.e. expected forward degree of nodes near the edges will fall sharply.

Now the only unknown variable in $(5)$ is $\overline{p_{c}}$ for which an expression is derived below. 


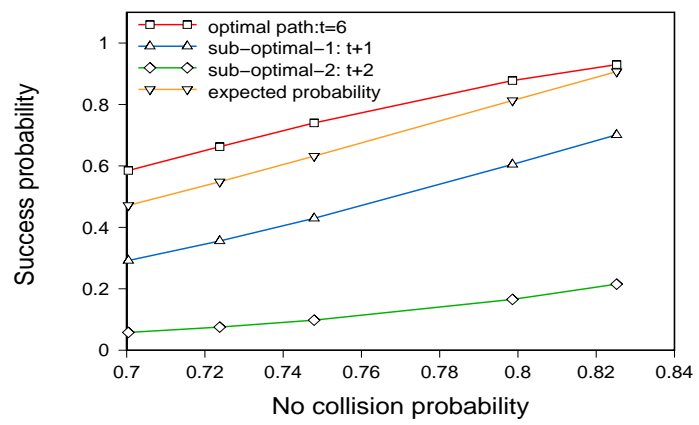

Fig. 2. Routing overhead and success probabilities of route discovery in BeeAdHoc.

\subsection{Collision Modeling}

As wireless channel is shared by mobile nodes, collisions are bound to happen. Having an 802.11b ad hoc network with its distributed coordination function (DCF) as MAC layer protocol, probability of collision is given by the following expression [11].

$$
p_{c}=1-\left(1-\frac{1}{C W_{\min }}\right)^{M-1}
$$

where $C W_{\min }(=31$ for $802.11 \mathrm{~b})$ is the minimum contention window and $M$ represents the number of contending nodes. Each of the $d_{a v g}$ neighbors may be contending for the channel access under heavy traffic. However, minimum contention case is the one in which a source initiates a route discovery process. Each node in this case is not a potential rebroadcasting node. Keeping in mind the concept of forward degree, $M=d_{a v g}-d_{f}-2$. Therefore, using (7), the probability of no collision is

$$
\overline{p_{c}}=\left(1-\frac{1}{C W_{\min }}\right)^{d_{\text {avg }}-d_{f}-2}
$$

(8) shows that $\overline{p_{c}}$ decreases exponentially with an increase in the number of nodes contending for the channel access.

\section{Route Optimality}

BeeAdHoc maintains multiple paths to a given destination. Each scout reaching the destination is likely to discover a new route. We do not consider the route caching behavior of BeeAdHoc in this model. Let us assume that there are $k$ edge disjoint paths between the source-destination pair with $t$ hops path as an optimal path. We further assume that a function $f[i-t]$ gives the total number of edge-disjoint paths of length $i$ between source-destination pair. 


\subsection{Probability of Optimal Path Discovery}

$B e e A d H o c$ discovers each link with a probability $\overline{p_{c}}$ and hence the probability of discovering an optimal path is $\epsilon=\left(\overline{p_{c}}\right)^{t-1}$. The probability of failure in finding an optimal path is then $(1-\epsilon)$. Now the probability of finding $j$ optimal paths out of a total of $f[0]$ optimal paths is simply a binomial distribution given as

$$
b(j ; f[0], \epsilon)=P(X[t]=j)=\left(\begin{array}{c}
f[0] \\
j
\end{array}\right) \epsilon^{j}(1-\epsilon)^{f[0]-j} .
$$

where $X[t]$ represents the number of $t$ hop paths discovered successfully. Using (9), the probability of discovering at least a single optimal path is

$$
P(X[t] \geq 1)=1-(1-\epsilon)^{f[0]},
$$

$(1-\epsilon)^{f[0]}$ is the probability of failure in discovering an optimal path which can be minimized either by increasing the value of $f[0]$ or $\overline{p_{c}}$. However, $f[0]$ can be increased by increasing node density $(\rho)$ which in turn reduces $\overline{p_{c}}$. Therefore, reducing the failure probability of route discovery is a tricky problem.

\subsection{Probability of Suboptimal Path Discovery}

Using (10), probability of discovering a suboptimal path of length $t+n$ hops where $n=1,2, \ldots, n$ is

$$
P(X[t+n] \geq 1)=1-\left(1-\epsilon\left(\overline{p_{c}}\right)^{n}\right)^{f[n]}
$$

where $f[n]$ is the number of edge-disjoint paths of length $t+n$ hops. Equation (11) shows that as $n \rightarrow \infty$, the probability of discovering a path of length $t+n$ hops approaches zero. Hence, suboptimal paths have a lower discovery probability as compared to optimal paths.

\subsection{Expected Probability of Path Establishment}

In addition to the path optimality problem, it is important to evaluate the marginal probability of path discovery. Using (10) and (11), the expected probability of finding a path (irrespective of the path length) is

$$
E\{X\}=\sum_{i=0}^{n} w[i]\left(1-\left(1-\epsilon\left(\overline{p_{c}}\right)^{i}\right)^{f[i]}\right)
$$

where $w[i]=\frac{f[i]}{k}$ is the normalized weight of the paths of lengths $i$. Expected probability of route discovery in sparse networks is close to 1 due to high value of $\overline{p_{c}}$. However, it substantially reduces in high density networks. We plotted (10), (11) and (12) (assuming $f[0] \gg f[1] \gg \ldots$ ) for varying values of $\overline{p_{c}}$ and the results are shown in Fig. 2. As the probability of no collision $\left(\overline{p_{c}}\right)$ rises, so 
does the probability of route discoveries. Second important observation is that as $\overline{p_{c}}$ approaches 1 , expected probability of route discovery equals the probability of optimal path discovery. Finally, we also note that optimal paths are more probable than suboptimal paths.

\section{Conclusion and Future Work}

In this paper, we developed mathematical models of two key performance metrics for BeeAdHoc protocol: routing overhead and route optimality. Routing overhead is a function of the expected forward degree, number of hops and collisions probability. We quantified the intuition that severe contention adversely affects the route optimality and may result in suboptimal route discovery. Route optimality, on the other hand, is a function of the collision probability, number of available paths and their corresponding distribution. We also conclude that the probability of discovering optimal paths is usually higher than the probability of discovering suboptimal paths. In future work we intend to derive an exact expression for expected forward degree and incorporate the mobility model.

\section{References}

1. Caro, G.D., Ducatelle, F., Gambardella, L.M.: AntHocNet: An adaptive natureinspired algorithm for routing in mobile ad hoc networks. Telecommunications (ETT), Special Issue on Self Organization in Mobile Networking 16(2) (2005)

2. Genes, M., Sorges, U., Bouazizi, I.: ARA - the ant-colony based routing algorithm for manets. In: Proceedings of ICPP Workshop on Ad Hoc Networks. (2002)

3. Farooq, M.: Bee-inspired Protocol Engineering: From Nature to Networks. Natural Computing Series. Springer ((In Press))

4. Wedde, H., Farooq, M., Pannenbaecke, T., Vogel, B., Mueller, C., Meth, J., Jeruschkat, R.: BeeAdHoc: an energy efficient routing algorithm for mobile ad hoc networks inspired by bee behavior. In: Proceedings of GECCO. (2005)

5. Rajagopalan, S., Shen, C.C.: ANSI: a swarm intelligence-based unicast routing protocol for hybrid ad hoc networks. Journal of System Architecture 52(8-9) (2006) 485-504

6. Kurkowski, S., Camp, T., Colagrosso, M.: MANET simulation studies: The incredibles. ACM SIGMOBILE Mobile Computing and Communications Review 9(4) (October 2005) $50-61$

7. Roth, M.: The markovian termite: A soft routing framework. In: Proceedings of IEEE Swarm Intelligence Symposium, SIS, April. (2007)

8. Saleem, M., Khayam, S.A., Farooq, M.: A formal performance modeling framework for bio-inspired ad hoc routing protocols. In: Proceedings of GECCO,. (2008)

9. Zahid, S., Shehzad, M., Ali, S.U., Farooq, M.: A comprehensive formal framework for analyzing the behavior of nature inspired routing protocols. In: Proceedings of IEEE Congress on Evolutionary Computing (CEC). (2007)

10. Bettstetter, C.: On the minimum node degree and connectivity of a wireless multihop network. In: Proceedings of MobiHoc,. (2002)

11. Heusse, M., Rousseau, F., Sabbatel, G.B., Duda, A.: Performance anomaly of 802.11b. In: Proceedings of IEEE INFOCOM. (2003) 